\title{
Metastatic small cell carcinoma of the prostate in an octogenarian with significant response to first-line chemotherapy
}

\author{
Anthony Wood ${ }^{1}$, Nabil Adra ${ }^{1 *}$, Liang Cheng ${ }^{2}$ and Roberto Pili ${ }^{1}$ \\ ${ }^{1}$ Division of Hematology \& Medical Oncology - Melvin \& Bren Simon Cancer Center, Indiana University School of Medicine, Indianapolis, Indiana, USA \\ ${ }^{2}$ Department of Pathology and Laboratory Medicine, Indiana University School of Medicine, Indianapolis, Indiana, USA
}

\section{Introduction}

Patients with small cell carcinoma of the prostate generally present with metastatic disease and have an estimated life expectancy measured in months. In this brief report, we present a case of rapidly progressive metastatic small cell carcinoma of the prostate in an octogenarian with significant response to split-dosed cisplatin and etoposide. This case report shows that cautious treatment with platinum-based regimens should still be considered in elderly patients given the potential for improvement in performance status and quality of life with chemotherapy. Additionally, this case highlights that platinum-based regimens are still considered the therapeutic backbone of prostate cancers with a mixed small cell carcinoma and adenocarcinoma phenotype.

\section{Case report}

An 81-year-old man with a history of Gleason $3+3$ adenocarcinoma of the prostate diagnosed in 2006 and actively monitored with an annual prostate specific antigen (PSA) and digital rectal exam presented to the emergency department in March of 2016 with 1 month of burning rectal pain. A CT scan demonstrated a 10.5 x 6.8 $\mathrm{x} 8.1 \mathrm{~cm}$ fungating mass obscuring the prostate. Biopsy of the mass showed a CD56+/TTF1+/synaptophysin+/PSA-/PSAP-/ps501-/ERGhyperchromatic high grade malignancy with nuclear molding necrosis and a high nuclear to cytoplasmic ratio consistent with small cell carcinoma (SCC) of the prostate (Figure 1). His PSA was $0.531 \mathrm{ng} / \mathrm{mL}$ at that time (Figure 2). He was started on chemotherapy with cisplatin and etoposide in May of 2016, but he experienced significant urinary retention and hematuria during cycle 1 which led to halting of therapy. In efforts to palliate his symptoms, he received 63 Gy of radiation over 35 fractions to his pelvis with significant relief of his rectal pain. Repeat CT scan in October of 2016 showed interval decrease of the prostatic mass to 2.7 x $5.9 \mathrm{~cm}$ (Figure 3A). In November of 2016, he developed right axillary and pectoral swelling. CT scan of the chest revealed a $13.1 \times 7.2 \mathrm{~cm}$ right-sided, pleural-based mass invading the chest wall and causing destruction of the fifth rib with associated precarinal, subcarinal, perihilar, and axillary lymphadenopathy. Biopsy of the axillary lymph node showed small cell carcinoma identical to that seen in the prostate biopsy. PSA remained low at $0.176 \mathrm{ng} / \mathrm{mL}$. The decision was made to restart chemotherapy in an attempt to improve functional status and quality of life. Given the patient's advanced age and prior adverse event with chemotherapy, he was restarted on split-dose cisplatin $30 \mathrm{mg} / \mathrm{m}^{2}$ and etoposide $100 \mathrm{mg} / \mathrm{m}^{2}$, both administered on days 1-2 of 28-day cycles with a plan to complete 6 total cycles. Repeat
CT scans following his second cycle of chemotherapy showed stable disease in his prostate with a significant reduction in size of his pleuralbased mass to $6.5 \times 1.9 \mathrm{~cm}$ (Figure 3B). To date, he has received 3 cycles of cisplatin and etoposide with significant continued response and no serious side effects.

\section{Discussion}

The prostate is one of the most common sites of extrapulmonary
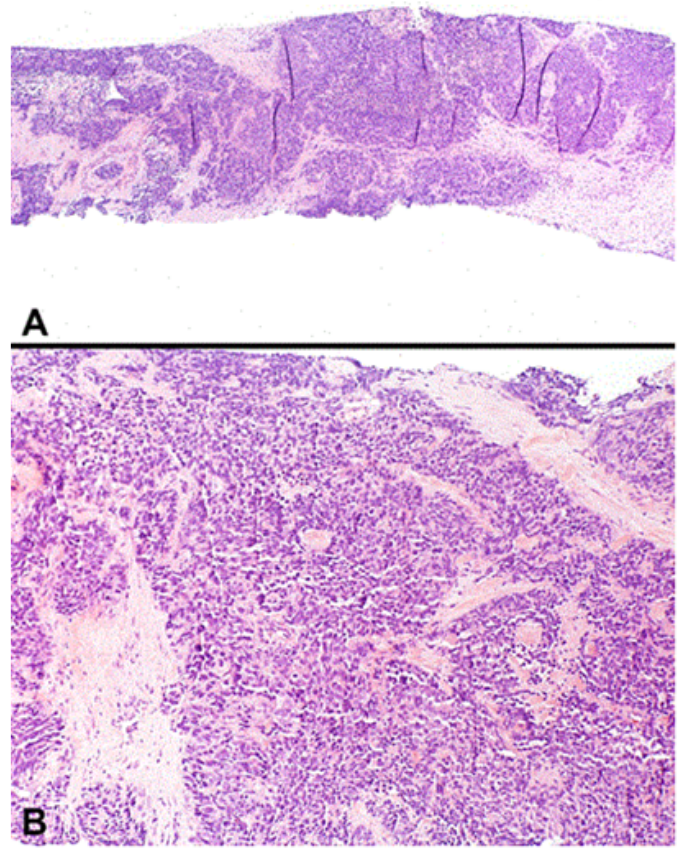

Figure 1. Small cell carcinoma (SCC) of the prostate (A and B hematoxylin \& eosin, A: original magnification x100; B: original magnification $\mathrm{x} 200)$.

Correspondence to: Nabil Adra, Division of Hematology \& Medical Oncology - Melvin \& Bren Simon Cancer Center, Indiana University School of Medicine, Indianapolis, Indiana, USA, Tel: 317-220-7786; Fax: 317-948-9302; Email: nadra@iu.edu

Key words: small cell carcinoma; prostate; chemotherapy; elderly; octogenarian

Received: February 17, 2017; Accepted: March 04, 2017; Published: March 07, 2017 


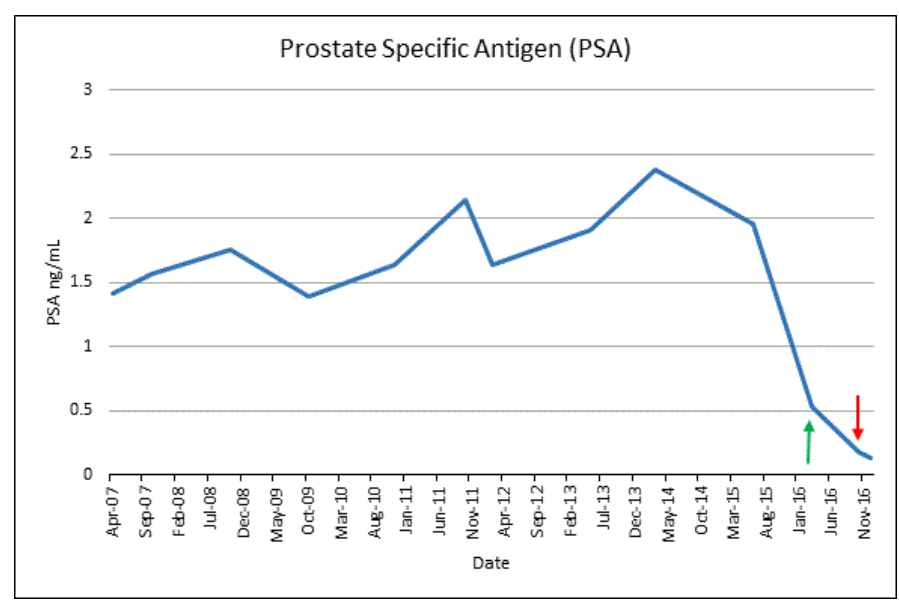

Figure 2. Prostate specific antigen (PSA) levels over time. Green arrow: PSA level at time of prostatic SCC diagnosis. Red Arrow: PSA level at time of metastatic progression following radiation therapy.

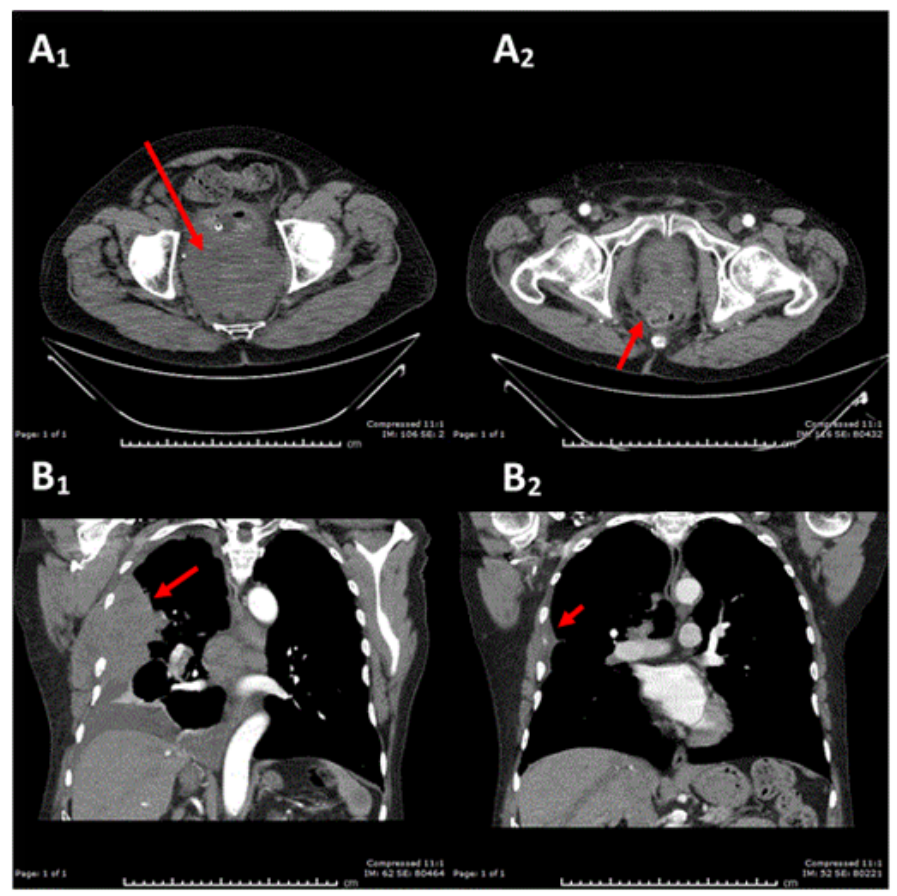

Figure 3. Top Row: CT scan of the pelvis demonstrating SCC of the prostate prior to radiation therapy (Figure $3 \mathrm{~A}_{1}-10.5 \times 6.8 \times 8.1 \mathrm{~cm}$ ) and following radiation therapy (Figure $3 \mathrm{~A}_{2}-2.7 \times 5.9 \mathrm{~cm}$ ). Bottom Row: CT scan of the chest demonstrating metastatic SCC of the prostate prior to chemotherapy (Figure $3 \mathrm{~B}_{1}-13.1 \times 7.2 \mathrm{~cm}$ ) and following 2 cycles of split-dose cisplatin/etoposide (Figure $3 \mathrm{~B}_{2}-6.5 \times 1.9 \mathrm{~cm}$ ).

neuroendocrine carcinomas [1]. Neuroendocrine tumors of the prostate include conventional adenocarcinomas with focal differentiation, carcinoid tumors, tumors with Paneth cell-like neuroendocrine differentiation, large cell neuroendocrine carcinomas, and small cell carcinomas $[1,2]$. While focal neuroendocrine differentiation is identified in most prostatic adenocarcinomas, SCC of the prostate is a less commonly occurring entity seen in $0.5-2 \%$ of men diagnosed with prostate cancer. It is characterized by pronounced prostatic enlargement with a disproportionately low PSA, unresponsiveness to androgen deprivation therapy (ADT), and a rapidly progressive course with frequent visceral metastases and lytic bone lesions [2,3]. In descending order of frequency, men with prostatic SCC most commonly present with voiding, neurologic (confusion, sensory/motor deficits), and/or constitutional symptoms [3]. SCC of the prostate arises in men with pre-existing adenocarcinoma who have received androgen deprivation in nearly half of cases $[2,4,5]$. This is believed to occur either due to adaptive dedifferentiation of malignant cells under the influence of $\mathrm{ADT}$ or the selective pressures of $\mathrm{ADT}$ favoring the proliferation of androgen receptor negative neuroendocrine cells [4].

The diagnosis of prostatic SCC can be made without tissue if the patient's clinical presentation is convincing and their functional status precludes therapeutic delays [2,6]. Histological examination of prostatic SCC is indistinguishable from SCC originating from other sites of the body. Pathological findings include rows of small cells with a high nuclear: cytoplasmic ratio, increased mitotic rates, coarse chromatin, necrosis, nuclear molding, and inconspicuous nucleoli [7]. Prostatic SCC can either be characterized as having a classical oat cell morphology (scant cytoplasm, open chromatin, small nuclei) or intermediate cell morphology (abundant cytoplasm, occasional nucleoli, large nuclei) [8]. Prostatic SCC's of the intermediate cell variant, in particular, can look morphologically similar to poorly differentiated Gleason 5 adenocarcinoma. Since these two entities have different therapeutic strategies, it is important to perform immunohistochemistry in all poorly differentiated specimens to identify the proper diagnosis [7]. A complete immunohistochemical panel including prostatic markers (PSA, PSAP, PSMA, ps501) and neuroendocrine markers (CD56, TTF-1, synaptophysin, and chromogranin) will be able to provide supplemental diagnostic information. Most prostatic SCCs will be negative or focally positive for prostatic markers, while poorly differentiated adenocarcinomas will be diffusely positive [8].

In situations such as the current case presentation where pathological specimens reveal small cell carcinoma in two separate sites of the body, it can be difficult to determine which region of the body the primary arose from. It is necessary to combine immunohistochemistry with clinical presentation to facilitate making an accurate diagnosis. Any positivity with prostatic markers, regardless of intensity, is highly suggestive of prostatic origin. CD44 and ERG are particularly specific for tumors of prostatic origin ${ }^{8}$. However, most prostatic SCC's are pan-negative for prostatic markers, which makes prostatic marker expression an unreliable discriminatory tool [8]. In addition, up to $50 \%$ of prostatic SCC's are TTF-1 positive, making this cellular marker unreliable in differentiating between pulmonary and prostatic primaries as well [9]. In this patient's case, the prostate was identified as the primary based on the temporal progression of clinical symptoms given that the immunohistochemical analysis was non-specific.

SCC of the prostate is an aggressive malignancy associated with a poor prognosis. An analysis of 191 cases of prostatic SCC registered in the Surveillance, Epidemiology, and End Results (SEER) database between 1973 and 2003 showed a median survival of 11 months, while patients over the age of 75 had a median survival of only 6 months [10]. The majority of patients present with metastatic disease. The most common sites of metastases are the bones, retroperitoneal lymph nodes, liver, lungs, and brain [4]. Therapeutic strategies are largely derived from experience with small cell lung cancer and retrospective reviews as data from randomized controlled trials with prostatic SCC is limited. Localized control with either a prostatectomy or radiation therapy should be considered in patients with limited stage disease as it has been associated with improved outcomes in retrospective reviews $[10,11]$. For patients who present with advanced disease, systemic chemotherapy with a platinum-based agent combined with either etoposide or docetaxel is the mainstay of therapy [6]. Radiotherapy can 
be used as needed for palliation of symptoms [2].

\section{Conclusion}

In this report, an octogenarian with rapidly progressive metastatic SCC of the prostate had a significant response to split-dosed cisplatin and etoposide combination chemotherapy. This case report shows that cautious treatment with platinum-based regimens should still be considered in elderly patients given the potential for improvement in performance status and quality of life with chemotherapy. Furthermore, patients with mixed SCC and adenocarcinoma of the prostate should still receive platinum-based regimens as first-line therapy, although androgen deprivation therapy is sometimes given concurrently depending on the phenotype of the mixed disease [2]. Despite our patient's excellent response, the course of the malignancy is such that he will likely relapse. While there is no effective second-line therapy established currently, novel agents being investigated in patients with neuroendocrine prostate cancer, such as aurora kinase A (AURKA) and poly(ADP-ribose) polymerase 1 (PARP-1) inhibitors, might have promising activity in this patient population whose therapeutic options are limited [12-15].

\section{Acknowledgements}

None

\section{Conflict of interest statement}

All authors have no conflicts of interest to disclose.

\section{References}

1. Priemer DS, Montironi R, Wang L, Williamson SR, Lopez-Beltran A, et al. (2016) Neuroendocrine Tumors of the Prostate: Emerging Insights from Molecular Data and Updates to the 2016 World Health Organization Classification. Endocr Pathol 27: 123-135.

2. Nadal R, Schweizer M, Kryvenko ON, Epstein JI, Eisenberger MA (2014) Small cell carcinoma of the prostate. Nat Rev Urol 11: 213-219.[Crossref]

3. Palmgren JS, Karavadia SS, Wakefield MR (2007) Unusual and underappreciated: small cell carcinoma of the prostate. Semin Oncol 34: 22-29.[Crossref]
4. Spiess PE, Pettaway CA, Vakar-Lopez F, Kassouf W, Wang X, et al. (2007) Treatment outcomes of small cell carcinoma of the prostate: a single-center study. Cancer 110: 1729-1737.[Crossref]

5. Wang HT, Yao YH, Li BG, Tang T, Chang JW, et al. (2014)Neuroendocrine prostate cancer (NEPC) progressing from conventional prostatic adenocarcinomas: factors associated with time to development of NEPC and survival from NEPC diagnosis - a systematic review and pooled analysis. J Clin Oncol 32: 3383-3390.

6. Aparicio AM, Harzstark AL, Corn PG, Wen S, Araujo JC, et al. (2013) Platinum-based chemotherapy for variant castrate-resistant prostate cancer. Clin Cancer Res 19: 36213630.[Crossref]

7. Wang L, Davidson DD, Montironi R, Lopez-Beltran A, Zhang S, et al. (2015) Small cell carcinoma of the prostate: molecular basis and clinical implications. Histol Histopathol 30: 413-424.[Crossref]

8. Furtado P, Lima MV, Nogueira C, Franco M, Tavora F (2011) Review of small cell carcinomas of the prostate. Prostate Cancer 2011: 543272.[Crossref]

9. Wang W, Epstein JI (2008) Small cell carcinoma of the prostate. A morphologic and immunohistochemical study of 95 cases. Am J Surg Pathol 32: 65-71.[Crossref]

10. Deorah S, Rao MB, Raman R, Gaitonde K, Donovan JF (2012) Survival of patients with small cell carcinoma of the prostate during 1973-2003: a population-based study. BJU Int 109: 824-830.[Crossref]

11. Weiner AB, Patel SG, Richards KA, Szmulewitz RZ, Eggener SE(2014) Populationbased analysis of treatment modalities and survival for clinically localized small-cell carcinoma of the prostate. Prostate Cancer Prostatic Dis 17: 286-291.

12. Melichar B, Adenis A, Lockhart AC, Bennouna J, Dees EC, et al. (2015) Safety and activity of alisertib, an investigational aurora kinase A inhibitor, in patients with breast cancer, small-cell lung cancer, non-small-cell lung cancer, head and neck squamouscell carcinoma, and gastro-oesophageal adenocarcinoma: a five-arm phase 2 study. Lancet Oncol 16: 395-405.

13. Monn MF, Cheng L (2016) Emerging trends in the evaluation and management of small cell prostate cancer: a clinical and molecular perspective. Expert Rev Anticancer Ther 16: 1029-1037.[Crossref]

14. Beltran H, Danila D, Montgomery B, et al. A phase 2 study of the aurora kinase inhibitor alisertib for patients with neuroendocrine prostate cancer (NEPC). Ann Oncol 27: LBA29.

15. Mateo J, Carreira S, Sandhu S, Miranda S, Mossop H, et al. (2015) DNA-Repair Defects and Olaparib in Metastatic Prostate Cancer. N Engl J Med 373: 1697-1708.[Crossref]

Copyright: (C2017 Wood A. This is an open-access article distributed under the terms of the Creative Commons Attribution License, which permits unrestricted use, distribution, and reproduction in any medium, provided the original author and source are credited. 